\title{
Achillea santolina and Raphanus sativus extracts downregulated NOTCH1, SIX1 and WNT1 developmental gene expressions in breast and colon cancer cell lines
}

\author{
Reham Mohasseb ${ }^{1}$, Reda Gaafar ${ }^{1}$, Mohamed Abdel Baseer ${ }^{2}$ and Mohamed L. Salem ${ }^{3}$ \\ ${ }^{1}$ Botany Department, Faculty of Science, Tanta University, Egypt \\ ${ }^{2}$ Botany and Microbiology, Faculty of Science, Al-Azhar University, Egypt \\ ${ }^{3}$ Zoology Department, Faculty of Science, Tanta University, Tanta, Egypt
}

Background: Although anticancer treatment with chemotherapy is effective, cancer response occurs due mainly to reduction of cancer stem cells. The development of cancer stem cell is regulated by several genes including NOTCH1, SIX1 and WNT1. Aim: we have found recently that treatment of Caco2, HepG2 and MCF-7 with Achillia santolina or Rahanus sativus results antitumor effect. In this study we aimed to evaluate the effect of these natural product on the gene expressions NOTCH1, SIX1 and WNT1 in Caco2 and MCF-7. Materials and Methods: Colon adenocarcinoma cell line (Caco2) and Breast cancer cell line (MCF-7) as well as normal amniotic cell line (WISH) were treated with 110 IC50 concentration of Achillea santolina and Raphanus sativus extracts to compared to 110 IC50 of cisplatin as a reference chemotherapy drug. Total RNA was extracted after 72 hours of treatment and incubation then CDNA was prepared for reverse transcriptase PCR for NOCH1, WNT1, and SIX1. Results: $R$. sativus treatment highly decreased NOTCH1 expression in MCF7 and Caco2 cell lines, while decreased SIX1 gene expression in Caco2 cell line and WNT1 in MCF7 cell line. On the other hand, A. santolina observably decreased the WNT1 gene expression in MCF7 cell line. Conclusion: The crude extracts of $R$. sativus decreased the developmental gene expression of NOTCH1, SIX1 in Caco2 cell line as same as on NOTCH1 and WNT1 in MCF-7 cell line more than cisplatin chemotherapy. While the A. santolina crude extract decrease the expression of WNT1 in MCF-7 cell line more than cisplatin chemotherapy.

Keywords: A. santolina; NOTCH1; R. sativus; SIX1; WNT1

Editor-in-Chief: Prof. M.L. Salem, PhD - Article DOI: 10.21608/JCBR.2021.62094.1175 